\title{
Urinary Smad1 as a New Biomarker for Diabetic Nephropathy in Patients with Type 1 Diabetes Mellitus
}

\author{
Mohamed Mohamed Ismail ${ }^{* 1}$, Sally kamal Ibrahim ${ }^{1}$ and Al Shaymaa Ahmed Ibrahim ${ }^{2}$ \\ ${ }^{1}$ Cairo University Kasr Alainy, Cairo, Egypt \\ ${ }^{2}$ Clinical and chemical pathogens' department, National Research Center, Egypt
}

*Corresponding authors: Mohamed Mohamed Ismail, Faculty of Medicine, Cairo University Kasr Alainy, Cairo, Egypt, Tel: 0201144479999; E-mail: mohamed_i_smail@yahoo.com

Received date: July 25, 2017; Accepted date: August 16, 2017; Published date: August 20, 2017

Copyright: ( 2017 Ismail MM, et al. This is an open-access article distributed under the terms of the Creative Commons Attribution License, which permits unrestricted use, distribution, and reproduction in any medium, provided the original author and source are credited.

\begin{abstract}
Introduction: Type 1 diabetes mellitus is an autoimmune disease affecting children worldwide with many consequences disturbing their quality of life. Diabetic nephropathy is among the most important complications of T1DM. The reliability of albuminuria as a predictor and prognosticator for renal injury has been frequently questioned. Many markers were investigated to replace urinary albumin. The current study aimed to assess the potential value of urinary Smad1 as a new biomarker for the early diagnosis and assessment of severity of diabetic nephropathy in children with.
\end{abstract}

Material and method: A case control cross sectional study with 53 patients with type 1 diabetes mellitus. 30 patients with diabetic nephropathy including 19 patients with microalbuminuria (urinary albumin creatinine ratio: $30-300 \mathrm{mg} / \mathrm{gm}$ ) and 11 patients with macroalbuminuria (urinary albumin creatinine ratio: $>300 \mathrm{mg} / \mathrm{gm}$ ). The remainder 23 patients had normal urinary albumin levels. In addition, there were 20 healthy age and sex matched children who served as control group. In all subjects we assessed urinary albumin, urinary creatinine and urinary albumin creatinine ratio and urinary Smad1 levels

Results: Urinary Smad1 levels and urinary Smad1 creatinine ratio show good sensitivity and specificity in detection of DN as compared to urinary albumin. The performance of SCR was better than urinary Smad1 with sensitivity and specificity of $100.0 \%$ and $96.0 \%$ for SCR, compared to sensitivity and specificity of $90.0 \%$ and $91.0 \%$ for urinary Smad1.

Conclusion: Urinary Smad1 is a promising new biomarker for detection of diabetic nephropathy in patients with type 1 diabetes mellitus with high sensitivity and specificity.

Keywords: Urinary Smad1; Biomarkers; Diabetic nephropathy

\section{Introduction}

Diabetes mellitus type 1 (T1DM) is an autoimmune disease affecting children worldwide with many consequences disturbing their quality of life. Diabetic nephropathy (DN) is among the most important complications of T1DM with significant association to patients' morbidity and mortality [1].

Currently, albuminuria is generally accepted as an early marker for detection of DN. However, reliability of albuminuria as a predictor and prognosticator for renal injury has been often questioned due to the fact that even patients with normal urinary albumin levels can suffer substantial pathological injury [2] or reduced glomerular filtration rate (GFR) [3]. In addition micro albuminuria, as an early marker for diabetic nephropathy, has numerous confusing concerns related to the effect of exercise, urinary tract infection, acute illness and cardiac failure. Also, it is known that micro albuminuria and macro albuminuria can occur in non-diabetic subjects, signifying the nonspecificity of albuminuria as a predictor designed for early diagnosis of diabetic nephropathy [4]. This led to a continuous pursuit for other biomarkers to overcome this essential flaw.
In this context, many markers were investigated. Mehta et al. classified urinary biomarkers according to the mechanism of their secretion in urine into markers of increased glomerular permeability including albumin, Cystatin C, immunoglobulins and transferrin; markers of extracellular matrix including transforming growth factorbeta (TGF- $\beta$ ), connective tissue growth factor (CTGF), Type IV collagen and Fibronectin; markers of epithelial-mesenchymal transition including fibronectin, matrix metalloproteinase and $\alpha$ smooth muscle actin; markers of tubulointerstitial damage including kidney injury molecule-1 (KIM-1), neutrophil gelatinase-associated lipocalin (NGAL), Liver-type fatty acid-binding protein (L-FABP), retinol binding protein and monocyte chemoattractant protein-1 (MCP-1) [5]. In addition many other urinary biomarkers including urinary $\gamma$-glutamyl transferase, urinary alkaline phosphatase, urinary peptidome-based classifier and osteoinductive factor are being investigated [6-8].

Smad1 in human was known for the 1st time in human the TGF- $\beta /$ Smads signaling pathway. TGF- $\beta$ is an eminent factor the pathway of fibrous tissue formation in many tissues. It is greatly expressed in glomerular epithelium. It is also expressed in the renal tubular epithelium [7]. Multiple animal studies provided evidence that Smad1 play a pivotal role in the development of DN. This is mediated 
Page 2 of 5

by increasing synthesis of extracellular matrix via different pathways [9-11]. Smad group of proteins is considered the key factor in the regulation of TGF- $\beta$ mediating intracellular signaling that finally result in cumulative formation of collagen IV in the extracellular matrix (ECM) and consequently extracellular matrix expansion which represent the major change early in (DN) [9].

So, Li et al. suggested urinary Smad1 as a potential diagnostic parameter for $\mathrm{DN}$ in adult patients with type 2 diabetes mellitus [9]. The current study aimed to assess the potential value of urinary Smad1 as a new biomarker for the early diagnosis and assessment of severity of diabetic nephropathy in children with T1DM.

\section{Subject and Methods}

The present study is a case control cross-sectional study. It was conducted on 53 patients with T1DM. Patients were excluded if their condition was complicated with diabetic ketoacidosis, infection or associated with cardiovascular, inflammatory, neoplastic, hepatic or renal disorders rather than DN. In addition, there were 20 healthy age and sex matched children who served as control group.

Among diabetic patients 30 patients were classified as suspected DN based on the elevated levels of urinary albumin creatinine ratio (UACR). The remainder 23 patients had normal urinary albumin levels. Patients with suspected DN were divided into two groups according to the level of albuminuria: microalbuminuria group of patients including 19 patients (UACR: 30-300 mg/gm) and macroalbuminuria group including 11 patients (UACR: $>300 \mathrm{mg} / \mathrm{gm}$ ).

The studied patients were subjected to careful history taking, thorough clinical examination and laboratory investigations including fasting blood glucose, HbAlc, serum cholesterol and serum triglycerides. Assessment of renal functions was achieved using serum creatinine, urinary albumin, urinary creatinine and urinary albumin creatinine ratio (UACR). Urinary albumin creatinine ratio was calculated in a first-morning spot urine collection after instructing the patients with special precautions that may alter the results of UACR. Patients were instructed to avoid exercise, exposure to cold a day before the sample till they give the morning spot sample. Also, they were instructed to avoid medications and to postpone the sample if there is any acute illness or hyperglycemia exceeding $160 \mathrm{mg} / \mathrm{dl}$. Urinary Smad1 levels were measured using quantitative ELISA technique using the following kits: Human Mothers against decapentaplegic homolog-1, MAD homolog-1 ELISA Kit Catalog No: E0647h.

Data obtained from the present study were computed using SPSS version 20. Continuous data were expressed in the form of mean \pm SD while categorical data were expressed in the form of number and percent. Comparison of continuous data was performed utilizing one way ANOVA, while categorical data were done using Chi-square test. Relation between variables were investigation by Pearson's correlation coefficient. $\mathrm{P}$ value less than 0.05 was considered statistically significant.

\section{Results}

Comparison between the studied groups regarding the clinical and laboratory data was illustrated in Table 1 .

\begin{tabular}{|c|c|c|c|c|c|}
\hline & All patients $n=53$ & Normoalbuminuria $n=23$ & Microalbuminuria $n=19$ & Macroalbuminuria $n=11$ & Controls $n=20$ \\
\hline Age (years) & $11.1 \pm 2.6$ & $11.5 \pm 2.6$ & $10.6 \pm 2.7$ & $11.3 \pm 3.4$ & $11.4 \pm 3.4$ \\
\hline BMI $\left(\mathrm{Kg} / \mathrm{m}^{2}\right)$ & $17.3 \pm 1.6$ & $17.4 \pm 2.1$ & $17.5 \pm 1.4$ & $16.7 \pm 1.0$ & $17.8 \pm 1.6$ \\
\hline $\operatorname{Sex}(M / F)$ & $(27 / 26)$ & $(11 / 12)$ & $(10 / 9)$ & $(6 / 5)$ & $(7 / 13)$ \\
\hline Duration of DM (years) & $6.0 \pm 2.1$ & $5.9 \pm 1.9$ & $6.4 \pm 2.4$ & $5.6 \pm 2.4$ & - \\
\hline Diabetic retinopathy $\mathrm{n}(\%)$ & $12(22.6)^{\mathrm{a}}$ & $1(4.3)^{\mathrm{bc}}$ & $5(26.3)$ & $6(54.5)$ & - \\
\hline $\mathrm{FBG}(\mathrm{mg} / \mathrm{dl})$ & $159.9 \pm 58.4^{\mathrm{a}}$ & $168.0 \pm 30.7^{\mathrm{ab}}$ & $135.0 \pm 70.2^{\mathrm{ac}}$ & $186.2 \pm 68.7^{\mathrm{a}}$ & $92.4 \pm 13.5$ \\
\hline HbA1c (\%) & $8.3 \pm 1.5^{\mathrm{a}}$ & $7.5 \pm 1.0^{\mathrm{abc}}$ & $8.8 \pm 1.6^{a}$ & $9.5 \pm 1.7^{\mathrm{a}}$ & $5.1 \pm 0.5$ \\
\hline Total cholesterol $(\mathrm{mg} / \mathrm{dl})$ & $156.4 \pm 47.2^{\mathrm{a}}$ & $131.4 \pm 38.0^{\mathrm{bc}}$ & $165.0 \pm 49.0^{\mathrm{ac}}$ & $193.8 \pm 31.1^{\mathrm{a}}$ & $135.0 \pm 26.0$ \\
\hline Triglycerides (mg/dl) & $109.9 \pm 40.6^{\mathrm{a}}$ & $99.9 \pm 33.9^{c}$ & $104.1 \pm 46.3^{\mathrm{ac}}$ & $140.8 \pm 28.8^{\mathrm{a}}$ & $80.7 \pm 18.0$ \\
\hline Serum creatinine (mg/dl) & $0.65 \pm 0.19$ & $0.62 \pm 0.19$ & $0.64 \pm 0.21$ & $0.72 \pm 0.14$ & $0.58 \pm 0.21$ \\
\hline Urinary albumin (mg/L) & $103.3 \pm 108.7^{a}$ & $11.7 \pm 8.2^{\mathrm{bc}}$ & $115.0 \pm 44.9^{\mathrm{ac}}$ & $274.4 \pm 76.8^{\mathrm{a}}$ & $7.3 \pm 4.1$ \\
\hline Urinary creatinine $(\mathrm{mg} / \mathrm{dL})$ & $111.0 \pm 51.6$ & $124.9 \pm 55.3^{c}$ & $113.3 \pm 53.5$ & $78.0 \pm 17.1^{\mathrm{a}}$ & $111.7 \pm 50.6$ \\
\hline UACR (mg/gm) & $118.1 \pm 133.8^{a}$ & $9.7 \pm 5.4^{\mathrm{bc}}$ & $115.2 \pm 45.0^{\mathrm{ac}}$ & $350.0 \pm 56.9^{\mathrm{a}}$ & $8.7 \pm 7.0$ \\
\hline Urinary smad1 (ng/ml) & $3.1 \pm 2.7^{\mathrm{a}}$ & $1.1 \pm 0.7^{\mathrm{bc}}$ & $3.2 \pm 1.6^{\mathrm{ac}}$ & $7.0 \pm 2.3^{a}$ & $0.5 \pm 0.2$ \\
\hline $\begin{array}{l}\text { Urinary smad } 1 / \text { creatinine } \\
\text { ratio }(\mathrm{SCR})(\mathrm{ng} / \mathrm{mg})\end{array}$ & $3.3 \pm 3.3^{a}$ & $0.8 \pm 0.5^{\mathrm{bc}}$ & $2.9 \pm 1.0^{\mathrm{ac}}$ & $9.0 \pm 2.3^{a}$ & $0.6 \pm 0.5$ \\
\hline
\end{tabular}


Citation: Ismail MM, Ibrahim SK, Ibrahim ASA (2017) Urinary Smad1 as a New Biomarker for Diabetic Nephropathy in Patients with Type 1

Table 1: Comparison between the studied groups regarding the clinical and laboratory data.

Of note, urinary Smad1 levels and SCR were significantly increased in all patients when compared with controls $(3.1 \pm 2.7$ vs. $0.5 \pm 0.2$ and $3.3 \pm 3.3$ vs. $0.6 \pm 0.5$ respectively) and in DN patients when compared with normoalbuminuric patients and healthy controls. Also, it was noted that urinary Smad1 levels and SCR were significantly increased in patients with macroalbuminuria when compared with those with microalbuminuria
In Table 2 urinary Smad 1 levels and urinary SCR showed significant correlation with $\mathrm{HbAlc}$, total cholesterol, triglycerides, urinary albumin and UACR while in DN patients, the significant correlations were only restricted to urinary albumin and UACR.

\begin{tabular}{|c|c|c|c|c|c|c|c|c|}
\hline & \multicolumn{4}{|c|}{ Urinary smad1 } & \multicolumn{4}{|c|}{ Urinary smad $1 /$ creatinine ratio } \\
\hline & \multicolumn{2}{|c|}{ All patients } & \multicolumn{2}{|c|}{ DN patients } & \multicolumn{2}{|c|}{ All patients } & \multicolumn{2}{|c|}{ DN patients } \\
\hline & $\mathbf{r}$ & $\mathbf{p}$ & $r$ & $\mathbf{p}$ & $\mathbf{r}$ & $\mathbf{p}$ & $\mathbf{r}$ & $\mathbf{p}$ \\
\hline Age (years) & -0.17 & 0.22 & -0.12 & 0.5 & -0.12 & 0.37 & -0.05 & 0.78 \\
\hline BMI $\left(\mathrm{Kg} / \mathrm{m}^{2}\right)$ & -0.09 & 0.5 & -0.15 & 0.4 & -0.13 & 0.34 & -0.23 & 0.2 \\
\hline Duration of DM (years) & -0.16 & 0.24 & -0.3 & 0.1 & -0.08 & 0.53 & -0.18 & 0.32 \\
\hline FBG (mg/dl) & 0.02 & 0.88 & 0.19 & 0.29 & 0.1 & 0.46 & 0.28 & 0.12 \\
\hline HbA1c (\%) & 0.35 & $0.009^{*}$ & 0.04 & 0.81 & 0.5 & $0.0001^{*}$ & 0.29 & 0.11 \\
\hline Total cholesterol (mg/dl) & 0.46 & $0.0004^{*}$ & 0.19 & 0.29 & 0.49 & $0.0001^{*}$ & 0.29 & 0.11 \\
\hline Triglycerides (mg/dl) & 0.33 & $0.014^{\star}$ & 0.24 & 0.19 & 0.36 & $0.006^{*}$ & 0.32 & 0.08 \\
\hline Serum creatinine $(\mathrm{mg} / \mathrm{dl})$ & 0.2 & 0.14 & 0.17 & 0.34 & 0.18 & 0.18 & 0.16 & 0.37 \\
\hline Urinary albumin (mg/L) & 0.87 & $0.0001^{*}$ & 0.77 & $0.0001^{*}$ & 0.83 & $0.0001^{*}$ & 0.69 & $0.0001^{*}$ \\
\hline Urinary creatinine $(\mathrm{mg} / \mathrm{dL})$ & -0.02 & 0.85 & 0.11 & 0.54 & -0.35 & $0.009^{\star}$ & -0.39 & 0.03 \\
\hline UACR (mg/gm) & 0.77 & $0.0001^{*}$ & 0.58 & $0.0006^{*}$ & 0.9 & $0.0001^{*}$ & 0.83 & $0.0001^{*}$ \\
\hline
\end{tabular}

Table 2: Correlation between urinary Smad1 levels and urinary SCR and the clinical and laboratory data in the studied patients.

The value of urinary Smad1 levels and urinary SCR in diagnosis of $\mathrm{DN}$ on was shown in Table 3. While both parameters showed good sensitivity and specificity, the performance of SCR was better with sensitivity and specificity of $100.0 \%$ and $96.0 \%$ compared to sensitivity and specificity of $90.0 \%$ and $91.0 \%$ for urinary Smad1.

\begin{tabular}{|l|l|l|}
\hline & smad1 & SCR \\
\hline Cut off & 2.05 & 1.67 \\
\hline AUC & 0.94 & 0.99 \\
\hline P & $0.0001^{*}$ & $0.0001^{*}$ \\
\hline Sensitivity & 0.9 & 1 \\
\hline Specificity & 0.91 & 0.96 \\
\hline
\end{tabular}

Table 3: Value of urinary Smad1 and SCR in the diagnosis of DN in the studied patients.
This high sensitivity and specificity was documented by the ROC curves for both smad1 and SCR in Figures 1 and 2 respectively. 


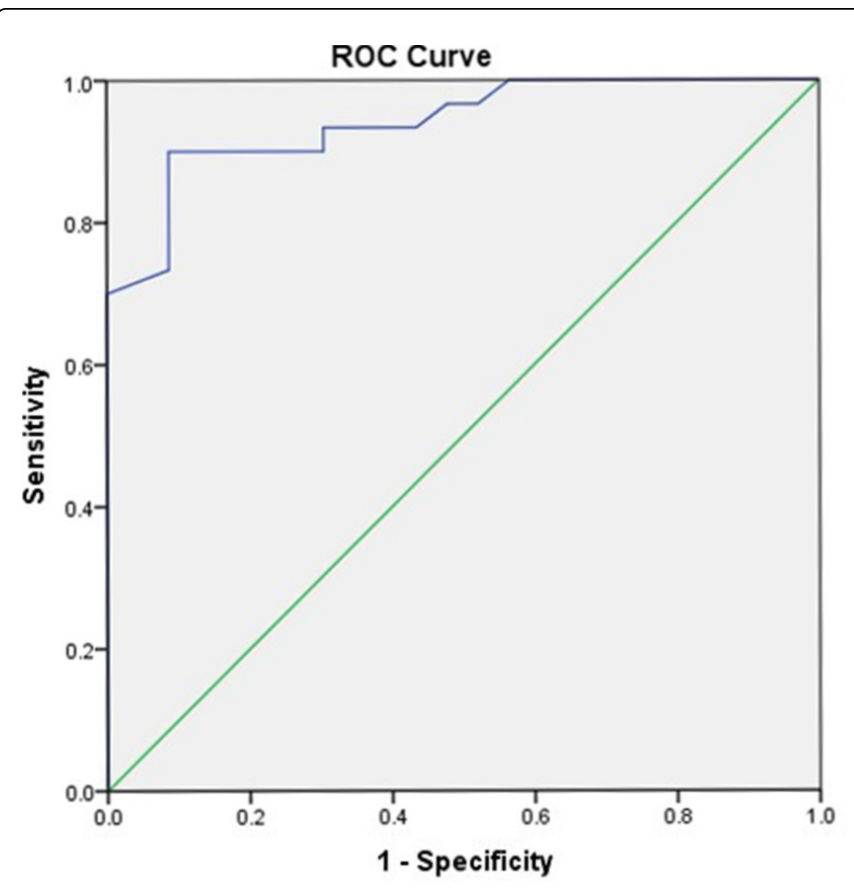

Diagonal segments are produced by ties.

Figure 1: ROC curve for urinary Smad1 as a biomarker for diabetic nephropathy.

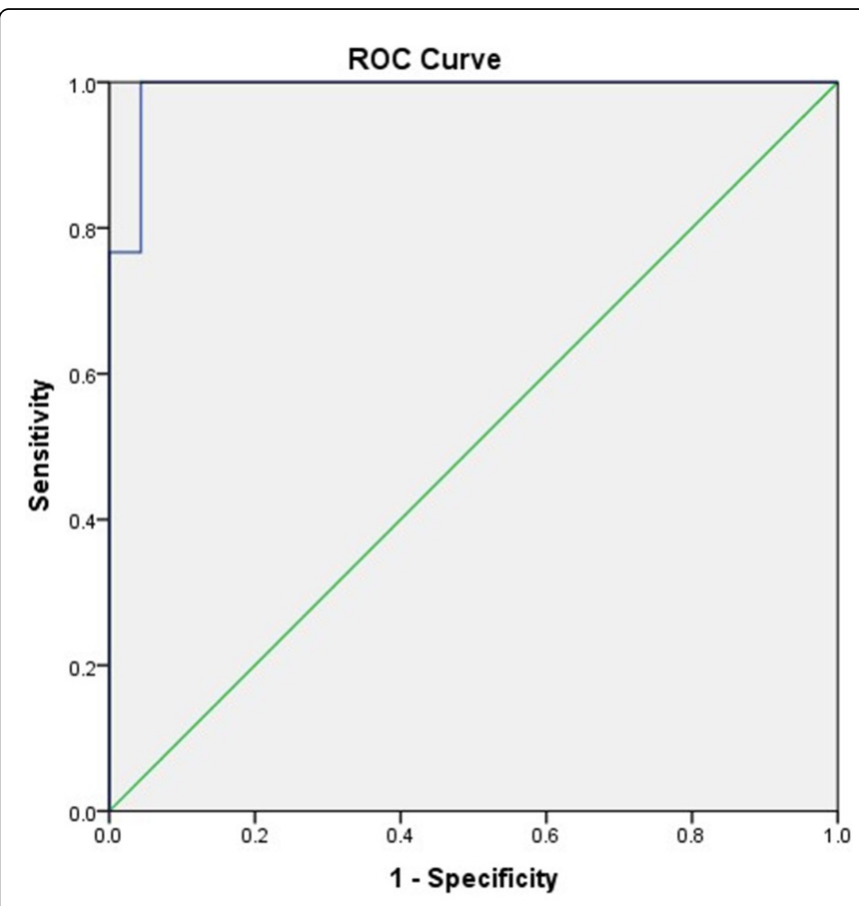

Figure 2: ROC curve for urinary SCR as a biomarker for diabetic nephropathy.

\section{Discussion}

The diagnosis and follow up of DN in children with T1DM is one of the most argumentary issues in pediatric nephrology. To the best of our knowledge, this study is the first to investigate the value of urinary Smad1 in the diagnosis of DN in children with T1DM. We provided a preliminary evidence that urinary Smad 1 and the derived urinary SCR can be useful tools in the diagnosis of DN. Moreover, levels of both parameters were successful indicators for the progression from microalbuminuria to macroalbuminuria. In addition, both urinary Smad1 and SCR correlated well with urinary albumin levels and UACR in $\mathrm{DN}$ patients.

These findings are in accordance with the study of Li et al., on adult patients with T2DM. Their study was the first ever to document the value of Smad1 and SCR in the diagnosis of DN in humans [9]. However, and in contrast to our study, they demonstrated significant direct correlation between SCR and diabetes duration. In another clue, the study of $\mathrm{Fu}$ et al., highlighted that high levels of urinary Smad1 were found in glomerular hyperfiltration (GHF) patients with T2DM $[12,13]$.

The suggestion of urinary Smad1 as a marker for diagnosis of DN is justified by its experimentally documented association with renal pathological changes prior to the clinical expression of albuminuria. The study of Matsubara et al., reported that the glomerular expression of Smad1 was significantly increased in diabetic rats with more mesangial matrix expansion under pathological examination. These finding were detected even in the absence albuminuria or glomerular hyperfiltration [10]. Moreover, Mima et al., noted that Urinary Smad1 of diabetic rats at 4 weeks was nicely correlated with mesangial matrix expansion at 24 weeks, while albuminuria showed a weaker association [11].

In a subsequent study, Tominaga et al., linked the increased expression of Smad1 in DN mice to role of bone morphogenetic protein-4 (BMP-4) which may act as an upstream regulatory molecule for the process of extracellular matrix accumulation in DN [14].

A more recent study by Matsubara et al., confirmed that BMP4/ Smad1 signaling pathway is the main conduit for the progression of mesangial expansion. Also, they suggested that blocking this signal could be a novel therapeutic strategy for diabetic nephropathy [15].

Nevertheless, and as Kato et al., commented [16], we should be careful about suggesting Smad1 as a biomarker for diagnosis and prediction of DN. This preliminary work with the limited number of patients and the cross-sectional design. Also, it is important to consider that the classification of DN in this study was based on urinary albumin level and not on renal histological changes. This work is only a step in the long road to develop a clinically reliable biomarker.

\section{Conclusions}

Urinary Smad1 is a promising new biomarker for detection of diabetic nephropathy in patients with type 1 diabetes mellitus with high sensitivity and specificity. Further wide scale studies are needed to confirm these results.

\section{Acknowledgement}

I deeply acknowledge my dear colleague Yomna shallan for her kind support. 
Citation: Ismail MM, Ibrahim SK, Ibrahim ASA (2017) Urinary Smad1 as a New Biomarker for Diabetic Nephropathy in Patients with Type 1 Diabetes Mellitus. J Diabetes Metab 8: 756. doi:10.4172/2155-6156.1000756

Page 5 of 5

\section{References}

1. Demirel F, Tepe D, Kara O, Esen I (2013) Microvascular complications in adolescents with type 1 diabetes mellitus. J Clin Res Pediatr Endocrinol 5: 145-149.

2. Krolewski AS, Niewczas MA, Skupien J, Gohda T, Smiles A, et al. (2014) Early progressive renal decline precedes the onset of microalbuminuria and its progression to macroalbuminuria. Diabetes Care 37: 226-234.

3. Caramori ML, Fioretto P, Mauer M (2003) Low glomerular filtration rate in normoalbuminuric type 1 diabetic patients: an indicator of more advanced glomerular lesions. Diabetes 52: 1036-1040.

4. Jain S, Rajput A, Kumar Y, Uppuluri N, Arvind A, et al. (2005) Proteomic Analysis of Urinary Protein Markers for Accurate Prediction of Diabetic Kidney Disorder. JAPI 53: 513-520.

5. Mehta S, Cabrera VJ, Upputalla R, Jim B (2013) Urinary biomarkers of diabetic nephropathy. Current Biomarker Findings 3: 67-78.

6. De Carvalho JA, Piva SJ, Hausen BS, Bochi GV, Kaefer M, et al. (2011) Assessment of urinary $\gamma$-glutamyl transferase and alkaline phosphatase for diagnosis of diabetic nephropathy. Clin Chim Acta 412: 1407-1411.

7. Siwy J, Schanstra JP, Argiles A, Bakker SJ, Beige J, et al. (2014) Multicentre prospective validation of a urinary peptidome-based classifier for the diagnosis of type 2 diabetic nephropathy. Nephrol Dial Transplant 29: 1563-1570.

8. Wang S, Wang Y, Zheng R, Zhao Z, Ma Y (2015) Osteoinductive factor is a novel biomarker for the diagnosis of early diabetic nephropathy. Int Clin Exp Pathol 8: 3110-3115.
9. Li Q, Feng L, Li J, Chen Q (2014) Urinary Smad1 is a new biomarker for diagnosis and evaluating the severity of diabetic nephropathy. Endocrine 46: 83-89.

10. Matsubara T, Abe H, Arai H, Nagai K, Mima A, et al. (2006) Expression of Smad1 is directly associated with mesangial matrix expansion in rat diabetic nephropathy. Lab Invest 86: 357-368.

11. Mima A, Arai H, Matsubara T, Abe H, Nagai K, et al. (2008) Urinary Smad1 is a novel marker to predict later onset of mesangial matrix expansion in diabetic nephropathy. Diabetes 57: 1712-1722.

12. Araoka T, Abe H, Tominaga T, Mima A, Matsubara T, et al. (2010) Transcription factor 7-like 2 (TCF7L2) regulates activin receptor-like kinase 1 (ALK1)/Smad1 pathway for development of diabetic nephropathy. Mol Cells 30: 209-218.

13. Fu WJ, Fang YG, Deng RT, Wen S, Chen ML, et al. (2013) Correlation of high urinary Smad1 level with glomerular hyperfiltration in type 2 diabetes mellitus. Endocrine 43: 346-350.

14. Tominaga T, Abe H, Ueda O, Goto C, Nakahara K, et al. (2011) Activation of bone morphogenetic protein 4 signaling leads to glomerulosclerosis that mimics diabetic nephropathy. J Biol Chem 286: 20109-20116.

15. Matsubara T, Araki M, Abe H, Ueda O, Jishage K, et al. (2015) Bone Morphogenetic Protein 4 and Smad1 Mediate Extracellular Matrix Production in the Development of Diabetic Nephropathy. Diabetes 64: 2978-2990.

16. Kato H, Si H, Hostetter T, Susztak K (2008) Smad1 as a biomarker for diabetic nephropathy. Diabetes 57: 1459-1460. 